\title{
Effects of heat and freeze on isolated erythrocyte submembrane skeletons
}

\author{
Ivan T. Ivanov ${ }^{1}$, Boyana K. Paarvanova ${ }^{1}$, Veselin Ivanov ${ }^{2}$, Kathrin Smuda ${ }^{3}$, Hans Bäumler ${ }^{3}$ \\ and Radostina Georgieva ${ }^{1,3}$
}

${ }^{1}$ Department of Physics and Biophysics, Medical Faculty of Thracian University, Stara Zagora 6000, Bulgaria

${ }^{2}$ Department of Chemistry and Biochemistry, Medical Faculty, Thracian University, Stara Zagora 6000, Bulgaria

${ }^{3}$ Institute of Transfusion Medicine, Charité-Universitätsmedizin Berlin, 10117 Berlin, Germany

\begin{abstract}
In this study we heated insoluble residues, obtained after Triton-X-100 $(0.1 \mathrm{v} / \mathrm{v} \%)$ extraction of erythrocyte ghost membranes (EGMs). Specific heat capacity, electric capacitance and resistance, and optical transmittance $(280 \mathrm{~nm})$ sustained sharp changes at $49^{\circ} \mathrm{C}\left(\mathrm{T}_{\mathrm{A}}\right)$ and $66^{\circ} \mathrm{C}\left(\mathrm{T}_{\mathrm{C}}\right)$, the known denaturation temperatures of spectrin and band 3 , respectively. The change at $\mathrm{T}_{\mathrm{A}}$ was selectively inhibited by diamide $(1 \mathrm{mM})$ and taurine mustard $(1 \mathrm{mM})$ while its inducing temperature was selectively decreased by formamide in full concert with the assumed involvement of spectrin denaturation. In the residues of EGMs, pretreated with 4,4'-diiso-thiocyanato stilbene-2,2'-disulfonic acid (DIDS), the change at $\mathrm{T}_{\mathrm{C}}$ was shifted from 66 to $78^{\circ} \mathrm{C}$ which indicated the involvement of band 3 denaturation. The freeze and rapid thaw of EGM residues resulted in a strong reduction of cooperativity of band 3 denaturation while the slow thaw completely eliminated the peak of this denaturation. These effects of freeze-thaw were prevented in residues obtained from DIDS-treated EGMs. The freeze-thaw of residues slightly affected spectrin denaturation at $49^{\circ} \mathrm{C}$ although an additional denaturation appeared at $55^{\circ} \mathrm{C}$. The results indicate preserved molecular structure and dynamics of the membrane skeleton in Triton-X-100 extracts of EGMs. The freeze-thaw inflicted strong damage on band 3 and spectrin-actin skeleton of EGM extracts which is relevant to cryobiology, cryosurgery and cryopreservation of cells.
\end{abstract}

Key words: Triton-X-100 shells - Spectrin - Band 3 - Freeze-thaw - Cryodamage

\begin{abstract}
Abbreviations: Diamide, diazenedicarboxylic acid bis( $N, N$-dimethylamide); DIDS, 4,4-diiso-thiocyanato stilbene-2,2'-disulfonic acid; DSC, differential scanning microcalorimetry; EGMs, erythrocyte ghost membranes; taurine mustard (taumustine), 2-[bis(2-chloroethyl)amino] ethanesulfonic acid; Triton-X-100, polyethylene glycol p-(1,1,3,3-tetramethylbutyl)-phenyl ether.
\end{abstract}

\section{Introduction}

The unique mechanical properties (deformability, elasticity and stability) of human erythrocytes are mainly due to the erythrocyte plasma membrane and the association of its membrane skeleton of peripheral proteins with its two major integral proteins, glycophorin and band 3 (AE1, the

Correspondence to: Ivan T. Ivanov, Department of Physics and Biophysics, Medical Faculty of Thracian University, Stara Zagora 6000, Bulgaria

E-mail: ivanov_it@gbg.bg anion exchanger) (Waugh and Agre 1988; Low et al. 1991). The membrane skeleton includes the third major protein of erythrocyte plasma membrane, spectrin, and a set of minor proteins such as actin, ankyrin, band 4.1, band 4.2, etc (Mohandas and Gallagher 2008). While the deformability of erythrocyte plasma membrane depends primarily on the state of spectrin-actin-glicophorin linkage, its mechanical stability chiefly depends on the linkage of spectrin via ankyrin to the tetrameric forms of band 3 (Van Dort et al. 1998; Beutler et al. 2000; Alberts et al. 2008) and on the interaction of spectrin with the negatively charged lipids within the internal monolayer of lipid bilayer (Hendrich et 
al. 1991; Michalak et al. 1994; Zwaal and Schroit 1997). The remaining portion of band 3 is in dimeric form and includes copies which freely difuse within the lipid bilayer or are linked to glycophorin (Tanner 1993; Reithmeier et al. 1996).

Erythrocyte shape stability and transformation are subject to a complex mechanism based on the minimum of the free energy of erythrocyte plasma membrane (Iglič 1997; Mukhopadhyay et al. 2002). The latter includes the elastic energy of membrane skeleton and the bending energy of lipid bilayer as well as the free energy of embedded integral proteins (Kralj-Iglič et al. 1996). This mechanism involves also the ratio of external to internal monolayer area which could be affected by the conformation of major band 3 protein (Gimsa and Ried 1995). Results of Hianik et al. (2000) demonstrated that the role of the membrane skeleton probably involves maintaining a higher compressibility of erythrocyte plasma membranes.

The plasma membranes of many nonerythroid cells of mammals and birds also contain similar anion exchanger protein (Alper 1991) and submembraneous spectrin-actin network (Mangeat 1988). In humans, impairment of erythrocyte deformability and elasticity has been correlated with many pathologic conditions, such as myocardial infarction, diabetes mellitus, essential hypertension, hereditary spherocytosis, sickle cell anemia, and malaria (Chien 1987; Simchon et al. 1987; Mokken et al. 1992; Ajmani 1997; Shelby et al. 2003; Delicou et al. 2015).

Hypotonically isolated human erythrocyte ghost membranes (EGMs) have been extensively studied with differential scanning calorimetry (DSC) which revealed several thermal denaturations of specific EGM proteins. The denaturation of spectrin, as represented by the A peak on the DSC thermogram, takes place at $49.5^{\circ} \mathrm{C}$ (Brandts et al. 1977). The cytoplasmic domain of band 3 protein denatures at $62^{\circ} \mathrm{C}$ (B2 peak), and the membrane domain of the band 3 protein denatures at $67^{\circ} \mathrm{C}$ (C peak) (Snow 1978).

A more simplified model of EGMs is frequently obtained by extracting EGMs with mild, polar detergents, typically Triton-X-100. This procedure releases the erythrocyte plasma membrane skeletons as insoluble residues, substantially delipidated and devoid of the most of membrane integral proteins (Sharma and Gokhale 2011). These, so called TritonX-100 shells (residues) contain chiefly spectrin, actin and the portion of band 3 protein comprising about $75 \%, 5 \%$ and $9 \%$, respectively, of their total residual protein (Lux et al. 1976). Other proteins of the Triton-X-100 shells include ankyrin (about 3\%), band 4.1 (about 5\%), and portions of band $4.2(2 \%)$. Triton X-shells preserve the shape and native two-dimensional supramolecular assembly of the membrane skeleton of parent erythrocytes (Yu et al. 1973). This is considered as evidence that membrane skeleton provides strong support for the shape and mechanical stability of human erythrocytes.
Recent studies (Ivanov et al. 2012; Ivanov and Paarvanova 2016) have noted changes in the dielectric properties (complex impedance, $Z^{*}$; complex capacitance, $C^{\star}$; dielectric loss curve) of human erythrocytes and their isolated impermeable EGMs and Triton-X-100 shells, induced at the spectrin denaturation temperature $\left(49.5^{\circ} \mathrm{C}\right)$. Based on the temperature dependence of the loss curve, the spectrin skeleton of EGMs was considered dielectrically active only at native state. Hence, the respective changes in $Z^{\star}$ and $C^{\star}$ at $49.5^{\circ} \mathrm{C}$ were considered as contribution of spectrin skeleton to the dielectric properties of native EGMs. Based on the strong frequency dependences of the changes in $Z^{*}$ and $C^{\star}$ at $49.5^{\circ} \mathrm{C}$, the methods of dielectric spectroscopy (Klösgen et al. 2011) revealed two dielectric relaxations on the membrane skeleton of EGMs. The first relaxation depended on the availability of linkages between the integral proteins and spectrin and was detected at such frequencies whereat the lipid bilayer did not allow penetration of the field into cytosole. It was present on erythrocytes and impermeable EGMs and not on Triton-X-100 shells. It was explained as a direct piezoeffect on the flexible spectrin filaments with a mechanical force originating from the frequency-dependent charging of lipid bilayer. The second relaxation was detected at higher frequencies allowing direct interaction of alternating field with the spectrin dipoles, presumably those formed on the triple-helical repeat units of spectrin monomers.

The present study is aimed at the exporation of Triton$\mathrm{X}-100$ shells of EGMs with emphasis on dielectric changes related to the thermal denaturations of their major proteins, spectrin and band 3 . These denaturations were detected by ultraviolet spectrophotometry and DSC. Thermal dielectroscopy detected two major dielectric relaxations of above mentioned second type, coupled to these denaturations. A study of these thermally-induced dielectric changes showed that freeze-thaw of shells altered the band 3 and spectrin, and the alternation of the former was more pronounced than that of the latter. The rapid thaw of frozen shells strongly modified the band 3 denaturation while the slow thaw resulted in its complete elimination.

\section{Materials and Methods}

\section{Materials}

DIDS (4,4'-diiso-thiocyanato stilbene-2,2'-disulfonic acid), $\mathrm{MgCl}_{2}, \mathrm{NaCl}$, phosphate buffer, diamide (diazenedicarboxylic acid bis( $N, N$-dimethylamide)), taurine mustard (2-[bis(2chloroethyl)amino] ethanesulfonic acid), Triton-X-100 (polyethylene glycol p-(1,1,3,3-tetramethylbutyl)-phenyl ether), EDTA (ethylenediaminetetraacetic acid) and formamide were purchased from Sigma Chemicals Co, St. Louis, MO, USA. 


\section{Isolation of erythrocytes}

Human blood samples were obtained from healthy donors (donor centres at Stara Zagora, Bulgaria and Charité, Berlin, Germany). Erythrocytes were isolated by centrifugation $(500 \times g, 5 \mathrm{~min})$ and three times washed in excess volume of cold $150 \mathrm{mM} \mathrm{NaCl}$ saline prior usage.

\section{Thermal stabilization of band 3 with DIDS}

DIDS is a membrane impermeable bifunctional covalent amino reagent, specifically binding and cross-linking band 3 monomer (Cabantchik and Greger 1992). Washed erythrocytes were suspended at hematocrit of 0.10 in $150 \mathrm{mM}$ $\mathrm{NaCl}$ saline, containing $5 \mathrm{mM}$ phosphate buffer, $\mathrm{pH} 7.8$, and $50 \mathrm{mM}$ DIDS at dark and at room temperature for $15 \mathrm{~min}$. The DIDS-treated cells were isolated, twice washed in excess volume of cold $150 \mathrm{mM} \mathrm{NaCl}$ saline to remove non-reacted DIDS. Under these conditions, more than 95\% of the DIDS resides on band 3 (Jennings and Passow 1979) resulting in strong thermal stabilisation of this protein and shifting of its denaturation temperature from $67^{\circ} \mathrm{C}$ to $80^{\circ} \mathrm{C}$ (Snow et al. 1978).

\section{Isolation of EGMs}

One volume of cold, dense suspension of washed erythrocytes (intact or DIDS-treated), hematocrit 0.60 , was rapidly and vigorously diluted in 15 volumes of $1^{\circ} \mathrm{C}$-cold hypotonic solution, containing $4 \mathrm{mM} \mathrm{MgCl}$ and $5 \mathrm{mM}$ phosphate buffer, $\mathrm{pH} 7.8$ and left at $4^{\circ} \mathrm{C}$ for $5 \mathrm{~min}$. The obtained leaky EGMs, used for Triton-X-100 extraction, were isolated by centrifugation $(4000 \times g, 12 \mathrm{~min})$ and additionally washed in the indicated hypotonic solution (Dodge et al. 1963).

\section{Preparation of Triton-X-100 shells of erythrocyte ghost membranes}

One ml of cold, leaky EGMs were vigourously mixed with equal volume of cold, washing solution $(10 \mathrm{mM} \mathrm{NaCl}$ saline, $4 \mathrm{mM}$ of $\mathrm{MgCl}_{2}, 5 \mathrm{mM}$ phosphate buffer, $\mathrm{pH}$ 7.8), that contained $0.2 \%(\mathrm{w} / \mathrm{v})$ Triton-X-100. When specially indicated, the EGMs were extracted at concentrations of Triton-X-100 higher than $0.1 \%$. The contact of membranes with detergent immediately turned their opaque suspension into a transparent medium. As the Triton-X-100 itself is a chelator of $\mathrm{Mg}^{2+}$, higher concentration ( $4 \mathrm{mM}$ ) of these ions were used. Phosphate buffer was prefered to Tris as Tris solubilises and disrupts the Triton-X-100 shells. The extraction of EGMs lasted $30 \mathrm{~min}$ at $4^{\circ} \mathrm{C}$. The obtained solution of crude, insoluble residues was diluted by 3 volumes of the indicated cold, washing medium and centrifuged $(8000 \times g$, $12 \mathrm{~min}$ ) to sediment the residues (Yu et al. 1973). The packed white paste of crude residues (about $0.2 \mathrm{ml}$ ) was immediately studied or, prior to study, additionally washed one, two or three times in excess volume of the washing medium. The term Triton X-100 shells (erythrocyte membrane skeletons, spectrin-actin skeletons) further means three-step washed shells, if not specifically indicated.

During the Triton-X-100 extraction of EGMs the residual protease activity could disrupt the proteins of membrane skeletons, as shown by Ciana et al. $(2005,2011)$. This protease activity originates from the white blood cell contamination of erythrocyte preparation. To reduce it at the stage of erythrocyte isolation, the buffy coat containing the majority of white blood cells, was carefully removed and the erythrocytes were thrice washed in excess volume of cold $150 \mathrm{mM}$ $\mathrm{NaCl}$ saline. The usage of EGMs, isolated after the hypotonic lysis of erythrocytes, additionally reduced protease activity.

\section{Differential scanning calorimetry of Triton-X-100 shells}

DSC measurements were performed using the DSC unit of a MSC Microcalorimeter, MicroCal, MA, USA. The measuring cell contained $1.442 \mathrm{ml}$ Triton-X-100 shells, diluted in the washing medium at a volume ratio of 1:0.3. The reference cell was filled with the washing medium. Scanning was carried out with a heating rate of $0.7^{\circ} \mathrm{C} / \mathrm{min}$ in the range from 20 to $90^{\circ} \mathrm{C}$. Only two portions of each sample were scanned if there was no substantial difference between the obtained thermograms.

\section{Spectrophotometric determination of thermal denaturations} in Triton-X-100 shells

$2.6 \mathrm{ml}$ of a solution containing $10 \mathrm{mM} \mathrm{NaCl}, 4 \mathrm{mM} \mathrm{Mg}^{2+}$ and $5 \mathrm{mM}$ phosphate buffer, $\mathrm{pH} 7.4$, was placed in a quartz glass cuvette and the optical transmittance at $280 \mathrm{~nm}\left(T_{280}\right)$ was set at $100 \%$. Thereafter $40 \mu$ of the Triton-X-100 shell paste was injected in the solution reducing $T_{280}$ to about $40 \%$. The optical transmittance was measured by a UV spectrophotometer (Milton Roy Spectronic 21D, USA), equipped with an electric heater of the measuring cuvette. Thermal denaturations were recorded heating the cuvette at a rate of $2^{\circ} \mathrm{C} / \mathrm{min}$ (Poklar et al. 1999). The temperature $t\left({ }^{\circ} \mathrm{C}\right)$ of the solution was measured by an electronic thermometer (accuracy $0.1^{\circ} \mathrm{C}$ ). The analog output signals of the spectrophotometer and the thermometer were both fed to a computer through a two-channel analog-to-digital converter.

The thermal denaturation of a test protein (fibrinogen) caused a sharp decrease in the optical transmitance as a result of the unfolding of its polypeptide chain and the change (typically decrease) in its molar volume. Spectrophtometric profile of the denaturation of fibrinogen (0.03 wt.\%), dissolved in $140 \mathrm{mM} \mathrm{NaCl}$ and $5 \mathrm{mM}$ phosphate buffer, $\mathrm{pH} 7.4$ 


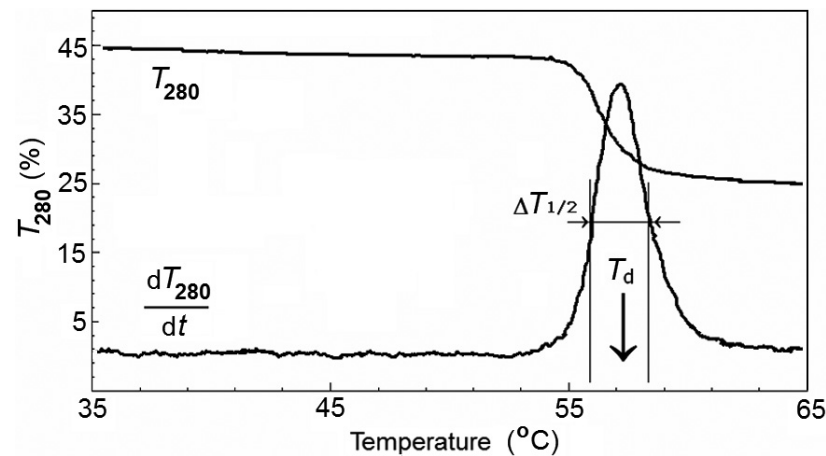

Figure 1. Temperature profile of the optical transmittance at $280 \mathrm{~nm}, T_{280}$ (above), and of the temperature derivative, $\mathrm{d} T_{280} /$ $\mathrm{d} t$ of $T_{280}$ (below), of fibrinogen solution. $T_{\mathrm{d}}$ and $\Delta T_{1 / 2}$ indicate the denaturation temperature and peak half-width of fibrinogen denaturation, respectively.

is shown in Figure 1. The obtained $T_{280} / t^{\circ} \mathrm{C}$ profile demonstrated the denaturation as a sigmoid decrease with midpoint at the temperature of denaturation, $T_{\mathrm{d}}$. The derivative curve, $\mathrm{d} T_{280} / \mathrm{d} t$, in Figure 1 corresponds to the rate of denaturation and specifys the top temperature of denaturation, $T_{\mathrm{d}}$, and the half-width, $\mathrm{D} T_{1 / 2}$, of the denaturation peak.

\section{Thermal dielectroscopy of Triton X-100 shells}

The sample cuvette (a cylindric glass tube with length $=$ $120 \mathrm{~mm}$, outer diameter $=4 \mathrm{~mm}$ and wall width $=0.5 \mathrm{~mm}$ ) contained two platinum wire electrodes mounted at a distance of about $4 \mathrm{~mm}$ from each other. Prior to heating, $70 \mu \mathrm{l}$ sample of the tested paste of Triton X-100 shells was placed in the cuvette and deaerated at room temperature under vacuum for $5 \mathrm{~min}$. The cuvette was put into a hole, drilled into an aluminum block. The block was heated with constant heating rate. During heating an alternating electric voltage of $80 \mathrm{mV}$ was imposed between the electrodes and the complex impedance, $Z^{\star}=Z_{\mathrm{re}}+\mathrm{j} Z_{\mathrm{im}}$, and complex capacitance, $C^{\star}=$ $C_{\mathrm{re}}+\mathrm{j} C_{\mathrm{im}}$, of the sample were continuously measured and separated into their real $\left(Z_{\text {re }}\right.$ and $\left.C_{\text {re }}\right)$ and imaginary $\left(Z_{\text {im }}\right.$ and $C_{\mathrm{im}}$ ) parts (Ivanov 2010). Here, $\mathrm{j}$ is the imaginary unit, $\mathrm{j}=(-1)^{0.5} \cdot Z^{\star}$ and $C^{\star}$ were measured in a sweep frequency mode at 12 frequencies between 0.05 and $10 \mathrm{MHz}$, scanned serially with integration time of $0.5 \mathrm{~s}$. The duration of each scan was less than $10 \mathrm{~s}$. The core instrument was a Solartron 1260 Impedance Analyzer (Schlumberger Instruments, Hampshire, England) interfaced to Toshiba PC using the Miniscan software.

Platinum electrodes, low sample conductance (about $40 \mu \mathrm{S})$, low electrode voltage $(80 \mathrm{mV})$ and frequencies above $50 \mathrm{kHz}$ were used in order to decrease electrode polarisation. The Impedance Analyzer removes the noise and harmonic distortions and has $0.1 \%$ accuracy and $0.001 \mathrm{~dB}$ resolution.

\section{Results}

Micrographs, taken with confocal microscope, showed the crude Triton-X-100 shells as pale mostly cup-shaped bodies with a diameter close to that of native erythrocytes (Fig. 2). Each shell was covered with a number of small vesicles adhering to it. The maximum diameter of these vesicles was $0.30 \mu \mathrm{m}$. Due to the low extracting concentration of the detergent, these vesicles could contain residual amounts of EGM lipids and integral proteins, mostly band 3.

\section{Thermal denaturation of spectrin and band 3 of Triton- -X-100 residuces}

Upon heating thrice washed Triton-X-100 shells the optical transmittance at $280 \mathrm{~nm}$ indicated two sigmoid changes with transition mid-temperatures at $T_{\mathrm{A}}\left(49^{\circ} \mathrm{C}\right)$ and $T_{\mathrm{C}}\left(66^{\circ} \mathrm{C}\right)$ (Fig. 3, lower curve). The temperature derivative of optical transmittance (Fig. 3, upper curve) exhibited the indicated changes as narrow peaks ( $\mathrm{A}$ and $\mathrm{C}$ ), centred at the above mentioned $T_{\mathrm{A}}$ and $T_{\mathrm{C}}$ temperatures. The narrow half-widths and high top temperatures of these peaks, compared to the denaturation peak of the test protein (Fig. 1), indicate involvement of protein denaturation in both events.

DSC scan of thrice washed Triton-X-100 shells exhibited two peaks of heat absorptions, centered at the same inducing temperatures, $T_{\mathrm{A}}\left(49^{\circ} \mathrm{C}\right)$ and $T_{\mathrm{C}}\left(66^{\circ} \mathrm{C}\right)$ (Fig. 4 , curve 1$)$. At the same inducing temperatures similar peaks have been calorimetrically obtained with samples of isolated EGMs and attributed to the heat denaturations of spectrin (the
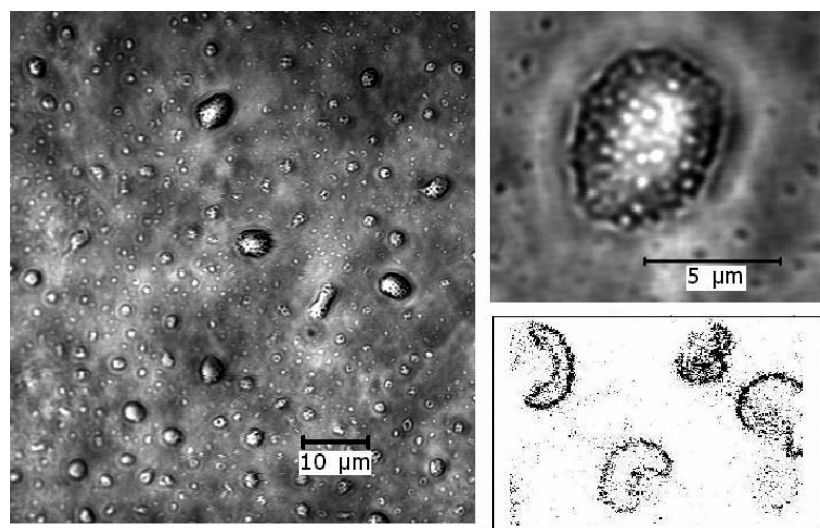

Figure 2. Micrographs of crude Triton-X-100 residues of EGMs. A general view (left) and an image of single Triton-X-100 shell at higher magnification in transmission mode (top-right). Confocal image of shells stained with the fluorescently labeled glycophorinspecific antibody CD235a-PE in fluorescence mode (bottom-right). Bar length reflects the magnification value. The images were taken with a confocal laser scanning microscope (LSM 510, Zeiss, Jena, Germany). 


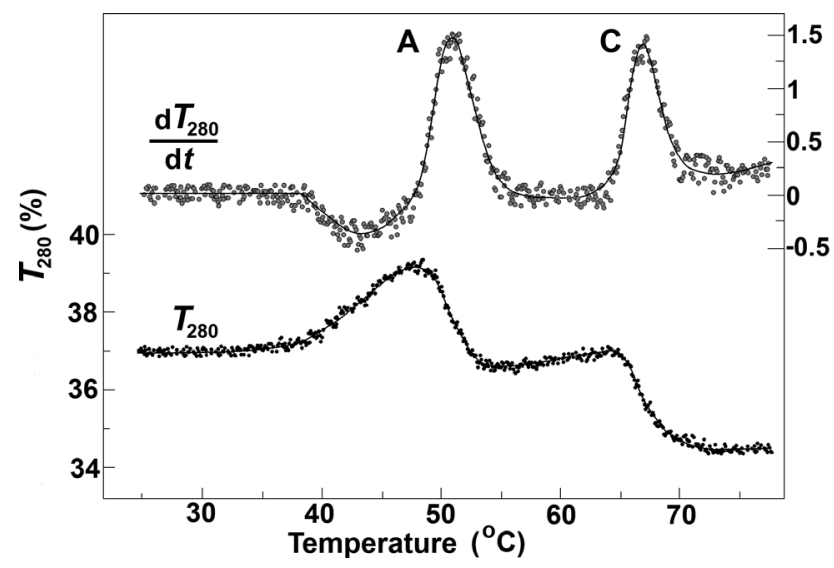

Figure 3. Temperature profile of the optical transmittance at $280 \mathrm{~nm}, T_{280}$ (below), and the temperature derivative of $T_{280}$, $\mathrm{d} T_{280} / \mathrm{dt}$ (above), of Triton-X-100 shells. Peaks A and C exhibit the heat denaturation of spectrin and anion exchanger, respectively.

calorimetric A peak) and the integral domain of band 3 (the calorimetric C peak), respectively (Brandts et al. 1977; Ivanov et al. 2007). Hence, it could be assumed that the peaks at the $T_{\mathrm{A}}$ and $T_{\mathrm{C}}$ top temperatures on the spectrophotometric (Fig. 3) and DSC (Fig. 4) thermograms of Triton-X-100 shells corresponded to the heat denaturations of spectrin and band 3 , respectively.

To additionally inspect these two denaturations, we subjected Triton-X-100 shell samples to continuous heating and measured the complex impedance and complex capacitance at various frequencies. At temperatures above $45^{\circ} \mathrm{C}$, two irreversible sigmoid changes were registered on the temperature profiles (thermograms) of the real and imaginary parts of these parameters, reflecting thermal damage to shells. For example, the changes on the thermogram of real capacitance, $C_{\text {re }}$, measured at $1 \mathrm{MHz}$, are shown in Fig. 5. The amplitudes of the two changes, $\Delta C_{\mathrm{A}}$ and $\Delta C_{\mathrm{C}}$ respectively, demonstrated remarkable frequency dependence (not shown). In order better to determine the inducing temperatures of these changes the temperature derivative of the sample capacitance $\left(\mathrm{d} C_{\mathrm{re}} /\right.$ $\mathrm{d} t$ ) was also obtained (Fig. 5). The inducing temperatures of these changes exactly coincided with above indicated denaturation temperatures, $T_{\mathrm{A}}$ and $T_{\mathrm{C}}$, respectively.

With once washed Triton-X-100 shells, the above indicated two sigmoid changes on the capacitance and resistance thermograms were centred at lower temperatures, $46^{\circ} \mathrm{C}$ and $55^{\circ} \mathrm{C}$, respectively (not shown). Increasing the number of washings to three, the mid-temperatures of these changes shifted upwards to the values, indicated in Fig. 5 and any additional wash did not produced effect on these temperatures. This result indicates that the residual amounts of Triton-X-100, remaining in the shells, reduced the above mentioned denaturation temperatures. The com- plete removal of Triton-X-100 resulted in a less pronounced temperature shift $\left(3^{\circ} \mathrm{C}\right.$, from 46 to $\left.49^{\circ} \mathrm{C}\right)$ of the first change and much more significant shift $\left(11^{\circ} \mathrm{C}\right.$, from 55 to $\left.66^{\circ} \mathrm{C}\right)$ of the second change. Thus, the effect of Triton-X-100 on the denaturation temperatures of spectrin and band 3 correlated the different ability of Triton-X-100 to bind and destabilize the peripheral and integral proteins of EGM, respectively.

The membrane skeletons of EGMs were also obtained after extraction of leaky EGMs with Triton-X-100 at the concentratons of detergent higher than $0.1 \%$. Typically $0.1 \%(\mathrm{v} / \mathrm{v})$ Triton-X-100 is sufficient to lyse erythrocytes, and even up to $0.4 \%$ concentrations will usually not harm most enzymes which are isolated. The sediment of insoluble residues, obtained at higher Triton-X-100 concentrations, had smaller volume. With increasing concentration of detergent from 0.2 to $0.4 \%$ the $\Delta C_{\mathrm{A}}$ and $\Delta C_{\mathrm{C}}$ amplitudes, as defined in Fig. 5, were reduced and at $0.8 \%$ these amplitudes became undetectably small (not shown). This result possibly indicates that EGM proteins became denatured by the detergent at the stage of extraction due to the high detergent concentration.

DIDS is a membrane-impermeable amino reagent, covalent inhibitor of band 3 of native erythrocytes. At low $(<50 \mu \mathrm{M})$ concentrations in ouside medium it specifically binds band 3, increasing its denaturation temperature stepwisely by $13^{\circ} \mathrm{C}$ (Snow et al. 1978). Using Triton-X-100 shells,

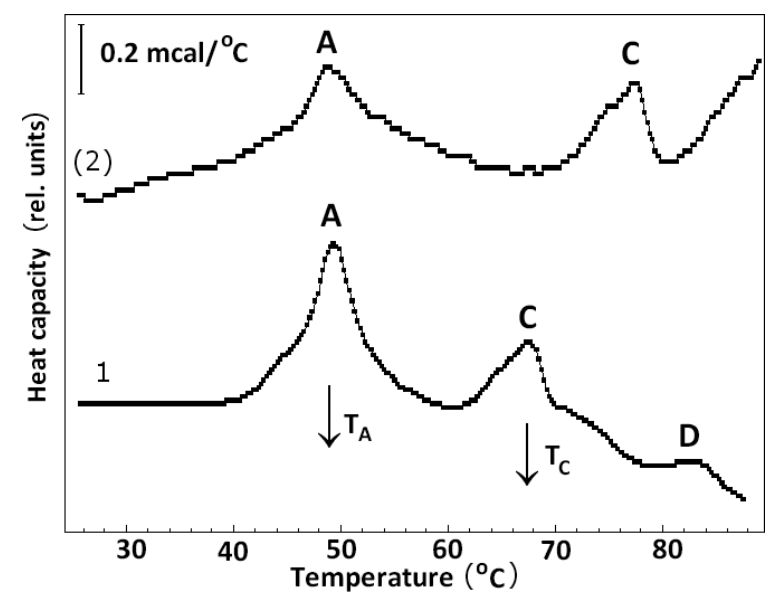

Figure 4. DSC thermograms of thrice washed Triton-X-100 shells of EGMs, isolated from intact erythrocytes (1) and from DIDS-treated erythrocytes (2). The A, C and D-peaks indicate the heat denaturation of spectrin, the anion exchanger and a peripheral protein (probably tropomyosin). $T_{\mathrm{A}}$ and $T_{\mathrm{C}}$ indicate the denaturation temperature of spectrin and anion exchanger, respectively. The suspension medium contained $4 \mathrm{mM} \mathrm{MgCl}_{2}$ and $150 \mathrm{mM} \mathrm{NaCl}$. Protein content and the heating rate were $3 \mathrm{mg} / \mathrm{ml}$ and $0.7^{\circ} \mathrm{C} / \mathrm{min}$, respectively. The under-membrane skeletons were released by extraction of EMs with $0.25 \%(\mathrm{v} / \mathrm{v})$ Triton-X-100. 


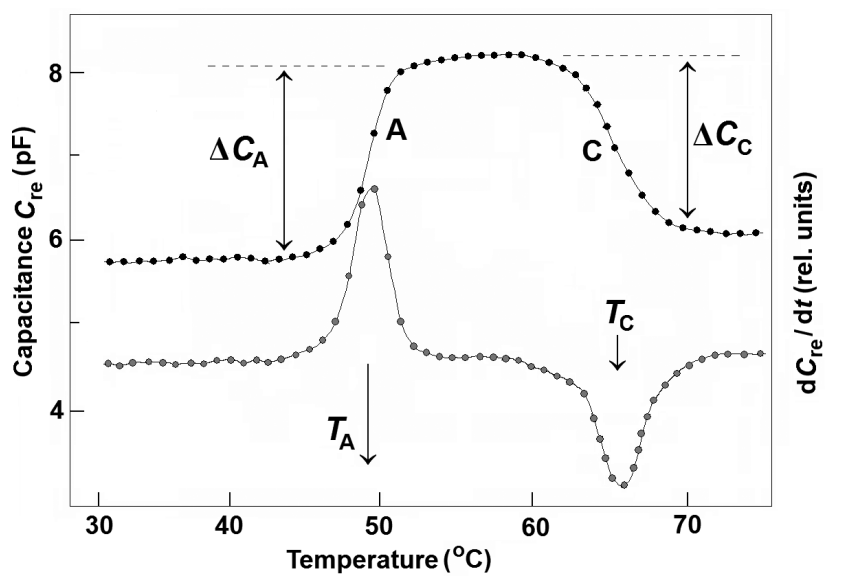

Figure 5. Temperature dependence of the capacitance, $C_{\mathrm{re}}(\bullet)$, and of the temperature derivative of the capacitance, $\mathrm{d} C_{\mathrm{re}} / \mathrm{d} t$ (o), of a sample of Triton-X-100 shells of EMs. The detergent-insoluble skeletons were prepared by extraction of EMs with $0.1 \%(\mathrm{v} / \mathrm{v})$ Triton-X-100. Amplitudes, $\Delta C_{\mathrm{A}}$ and $\Delta C_{\mathrm{C}}$, of the capacitance changes due to the denaturations of spectrin and band 3 at the mid temperatures $T_{\mathrm{A}}$ and $T_{\mathrm{C}}$, respectively, are shown. Arrows indicate the mid-temperatures of the sigmoid changes in sample capacitance. The washing medium contained $10 \mathrm{mM} \mathrm{NaCl}, 4 \mathrm{mM}$ $\mathrm{MgCl}_{2}$ and $8 \mathrm{mM}$ phosphate buffer, $\mathrm{pH}$ 7.8. Protein content, heating rate and frequency were $30 \mathrm{mg} / \mathrm{ml}, 2.0^{\circ} \mathrm{C} / \mathrm{min}$ and $1 \mathrm{MHz}$, correspondingly.

isolated from DIDS-treated erythrocytes, similar step-wise shift of $T_{\mathrm{C}}$ from $66^{\circ} \mathrm{C}$ to $78^{\circ} \mathrm{C}$ was registered on their DSC thermogram (Fig. 4, curve 2) and on their spectrophotometric, capacitance and resistance thermograms (not shown). At the same time, DIDS had no affect on the inducing temperature $\left(T_{\mathrm{A}}\right)$ of the first change. The same step-wise shift of $T_{C}$ was obtained adding DIDS (final concentration 50 $\mu \mathrm{M})$ to Triton X-shells, isolated from intact EGMs, during the first wash of shells. In conclusion, this specific threshold effect of DIDS on thermal stability of Triton-X-100 shells, strongly supports the involvement of band 3 denaturation in the change at $T_{\mathrm{C}}$.

Diamide is a bivalent sulfhydryl reagent while taurine mustard is a dialkylating agent both specifically cross-linking spectrin dimers, when applied at low $(1 \mathrm{mM})$ concentration on native erythrocytes (Fischer et al. 1978; Wildenauer et al. 1980). While it is not bound itself, diamide mediates the formation of disulfide bonds between spatially adjacent sulfhydryl groups primarily in spectrin. Both inter- and intra-molecular disulfide bonds are formed: about one per 30 spectrin dimers of the former type and one per three spectrin dimers of the latter type. In this study, we exploited the specific action of diamide and taurine mustard toward spectrin, adding these reagents to thrice washed Triton-X-100 shells at the final concentration of $1 \mathrm{mM}$. Both reagents inhibited about 3-fold stronger the amplitude of the sigmoid change at $T_{\mathrm{A}}$ compared to that at $T_{\mathrm{C}}$ (not shown), whereas the values of $T_{\mathrm{A}}$ and $T_{\mathrm{C}}$ temperatures were not affected. This result is in line with the assumed involvement of spectrin in the denaturation of Triton-X-100 shells at $T_{\mathrm{A}}$. In addition, this result indicated that specific cross-linking and resulting immobilisation of intramolecular segments of spectrin reduced the dielectric polarisation of Triton-X-100 shells.

Polar organic solvents are heavily used in various biomedical applications. For example, formamide lowers the melting temperatures of deoxyribonucleic acids linearly by $2.4-2.9^{\circ} \mathrm{C} / \mathrm{mol}$ of formamide (Blake and Delcourt 1996). This effect is used to accelerate the hybridization of deoxyribonucleic acids. Applied on native erythrocytes and impermeable EGMs, the great majority of polar organic solvents destabilised much stronger band 3 than spectrin (Ivanov 2001; Paarvanova et al. 2012). As an exception, formamide displayed a specific ability to destabilise spectrin, decreasing its denaturation temperature at about 3 time stronger rate compared to that of band 3 . In this study, formamide demonstrated even more its specific effect on the proteins of Triton-X-100 shells. At concentrations less than 7\% (v/v) it reduced linearly the $T_{\mathrm{A}}$ and $T_{\mathrm{C}}$. The former, however, was reduced at about a 10 -fold higher rate compared to the latter (not shown). This finding is in line with the assumed participation of spectrin in the denaturation at $T_{\mathrm{A}}$.

Added to the Triton-X-100 residues prior to heating, EDTA strongly reduced the amplitudes, $\Delta C_{\mathrm{A}}$ and $\Delta C_{\mathrm{C}}$, and decreased the inducing temperatures, $T_{\mathrm{A}}$ and $T_{\mathrm{C}}$, on the capacitance and resistance thermograms (not shown). For example, $5 \mathrm{mM}$ EDTA inhibited about three times the capacitance changes $\Delta C_{\mathrm{A}}$ and $\Delta C_{\mathrm{C}}$ (as defined in Fig. 3), and decreased the $T_{\mathrm{A}}$ and $T_{\mathrm{C}}$ by $4^{\circ} \mathrm{C}$ and $11^{\circ} \mathrm{C}$, respectively. This result is in line with the known ability of EDTA to disrupt the structure and solubilize the proteins of EGMs (McMillan and Luftig 1973).

\section{Dielectric relaxations on spectrin and band 3 of Triton- $X-100$ residues}

As noted above the amplitudes of detected changes at $T_{\mathrm{A}}$ and $T_{\mathrm{C}}$ on the complex impedance and capacitance thermograms of Triton-X-100 residues displayed strong frequency dependences. These frequency effects reflect the contribution of spectrin and band 3 to the dielectric polarisation of Triton-X-100 residues as the amplitudes of both dielectric changes at $T_{\mathrm{A}}$ and $T_{\mathrm{C}}$ were reduced to zero after the thermal denaturation of respective proteins. To inspect both frequency dependences in more detail, dielectroscopy methods were further applied. They allow get insight into the molecular mobility of electric dipoles associated to proteins involved in respective dielectric relaxations. In contrast to the measurement of sample impedance, the measurement of 
sample capacitance was impeded at frequencies, lower than $100 \mathrm{kHz}$ due to the electrode polarisation. Thermograms of complex impedance, $Z^{*}=Z_{\mathrm{re}}+\mathrm{j} Z_{\mathrm{im}}$, were therefore used to investigate the frequency dependences of the amplitudes of the dielectric changes at $T_{\mathrm{A}}$ and $T_{\mathrm{C}}$.

The amplitudes of the detected change in $Z_{r e}$ at $T_{A}$ were initially defined as $\Delta Z_{r e}=\left(Z_{r e}\right)_{\text {native }}-\left(Z_{r e}\right)_{\text {denatured }}$, where $\left(Z_{r e}\right)_{\text {native }}$ and $\left(Z_{r e}\right)_{\text {denatured }}$ are the real part of sample impedance at the native state of spectrin (at a temperature $3^{\circ} \mathrm{C}$ less than $T_{\mathrm{A}}$ ) and at the denatured state of spectrin (at a temperature $3^{\circ} \mathrm{C}$ greater than $T_{\mathrm{A}}$ ), respectively. This change, however, levels off within a substantial temperature interval of about $6^{\circ} \mathrm{C}$ where it is superimposed on the continuous, thermally induced change in the electrolyte conductivity. To compensate for the latter, which had no relation to the temperature-induced event at $T_{\mathrm{A}}$, the change in $Z_{\text {re }}$, taking place over an equal temperature interval prior the denaturation at $T_{\mathrm{A}}$, was likewise determined and subtracted from the initial $\Delta Z_{r e}$. Using the same algorithm the amplitude of the real changes of $Z_{\mathrm{im}}$ at $T_{\mathrm{A}}, \Delta Z_{i m}$, was determined and corrected for the thermally induced change in electrolyte conductivity. Also, the corrected amplitudes of the changes in $Z_{\mathrm{re}}$ and $Z_{\mathrm{im}}$ at $T_{\mathrm{C}}$ were likewise determined and further used.

Based on the theory of dielectroscopy (Kell 1987; Klösgen et al. 2011) the frequency dependences of corrected amplitudes $\Delta Z_{r e}$ and $\Delta Z_{i m}$ were studied plotting the $-\Delta Z_{i m} v s . \Delta Z_{r e}$ for each denaturation temperature, $T_{\mathrm{A}}$ and $T_{\mathrm{C}}$. Complex plane (Nyquist) plot of $-\Delta Z_{i m} v s . \Delta Z_{r e}$, determined at the spectrin denaturation temperature, $T_{\mathrm{A}}$, had the form of a semicircle arc, situated below the argument axis (Fig. 6).

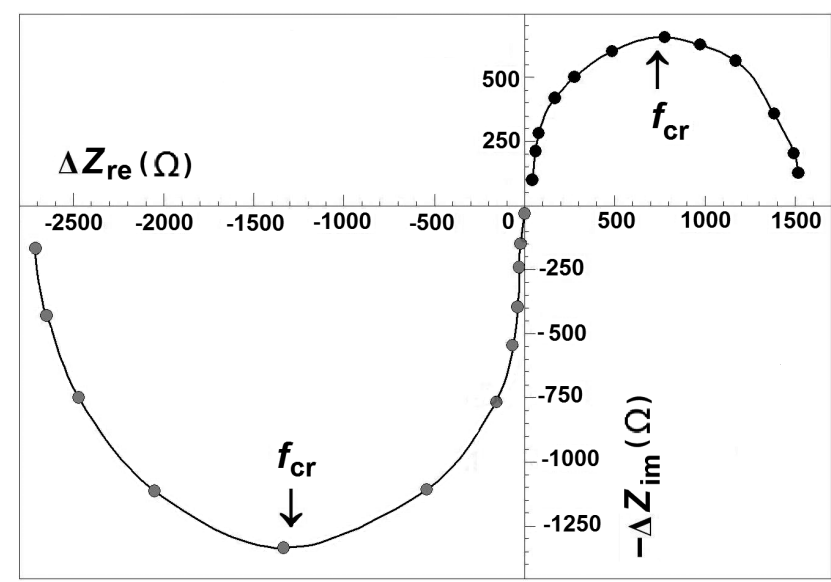

Figure 6. Complex plane plot of $-\Delta Z_{\mathrm{im}} v s . \Delta Z_{\mathrm{re}}$ at the denaturation temperature of spectrin (o) and of band $3(\bullet) . \Delta Z_{\text {re }}$ and $\Delta Z_{\text {im }}$ are the amplitudes of the changes of the real and imaginary parts of complex impedance, respectively, of packed Triton-X-100 shells at the indicated temperatures of denaturation. The critical frequencies of detected dielectric relaxations are indicated by $f_{\mathrm{cr}}$.
The complex plane plot of $-\Delta Z_{i m} v s . \Delta Z_{r e}$, determined at the band 3 denaturation temperature, $T_{C}$, represented another semicircle arc, situated above the argument axis (Fig. 6). According to Kell (1987) and Klösgen et al. (2011) each semicircle arc in Fig. 6 revealed a dielectric relaxation of Debye type, i.e. polarisation mechanism with a single critical frequency, $f_{\mathrm{cr}}$ (a single relaxation time, $\tau=1 /\left(2 \pi f_{\mathrm{cr}}\right)$ ).

As shown in Fig. 6, the $-\Delta Z_{i m} v s . \Delta Z_{r e}$ plot at the spectrin denaturation temperature revealed only one dielectric relaxation due to the direct interaction of electric field with the dipole moments of spectrin (Ivanov et al. 2012; Ivanov and Paarvanova 2016). This finding is consistent with the basic conception that Triton-X-100 residues lacked closed lipid bilayer encapsulating them. The critical frequency, $f_{\mathrm{cr}}$, for this relaxation is implicitly determined by the top point of the corresponding semicircle arc, indicated by arrow in Figure 6. From multiple experiments $(n=5)$ with shells, isolated and washed at $10 \mathrm{mM} \mathrm{NaCl}$ concentration this critical frequency was determined at $830 \mathrm{kHz}$ (Fig. 6). With shells, isolated at $10 \mathrm{mM} \mathrm{NaCl}$ and washed by media with increased $\mathrm{NaCl}$ concentration up to $120 \mathrm{mM}$ the critical frequency of spectrin relaxation linearly increased from $830 \mathrm{kHz}$ to $9 \mathrm{MHz}$ (not shown). The presence of glycerol, known for its ability to strongly increase the viscosity of solutions, linearly decreased the critical frequency of spectrin relaxation. For example, at $120 \mathrm{mM} \mathrm{NaCl}$, this critical frequency was decreased from $9 \mathrm{MHz}$ at zero \% glycerol to about $5 \mathrm{MHz}$ at $40 \%$ glycerol (not shown). These data are in full concert with the same dependences, recently reported for the spectrin skeleton of intact erythrocytes and impermeable EGMs (Ivanov and Paarvanova 2016) and indicate preserved molecular structure and dynamics of membrane skeleton in Triton-X-100 shells.

On the other hand the critical frequency, $\mathrm{f}_{\mathrm{cr}}$ for the $-\Delta Z_{\text {im }}$ vs. $\Delta Z_{\text {re }}$ plot at the denaturation temperature of band 3 was determined $1300 \mathrm{kHz}(n=5)$. It did not depend on the $\mathrm{NaCl}$ concentration $(10-100 \mathrm{mM})$ and on the presence of glycerol (not shown). Both critical frequencies were not affected by the preliminary DIDS-treatment of erythrocytes.

Thermal dielectroscopy was employed to investigate the alterations of major proteins, spectrin and band 3, induced by preliminary freeze-thaw of Triton-X-100 shells. In Figure 7 these alterations are demonstrated by the temperature derivative of the resistance, $Z_{\text {re }}$, measured at $100 \mathrm{kHz}$. A similar result was obtained using derivative thermograms of other components $\left(Z_{\mathrm{im}}, C_{\mathrm{re}}\right.$ and $\left.C_{\mathrm{im}}\right)$ of complex impedance, $Z^{\star}$, and capacitance, $C^{\star}$, measured at various frequencies between $100 \mathrm{kHz}$ and $5 \mathrm{MHz}$ (not shown). The above mentioned dielectric changes at $T_{\mathrm{A}}$ and $T_{\mathrm{C}}$ were altered in the residues subjected to preliminary freeze-thaw (Fig. 7, curves 2, 3 and 4), compared to control shells not subjected to subzero temperatures (Fig. 7, the curve 1). In addition, the frequency dependences of the amplitudes of respective 


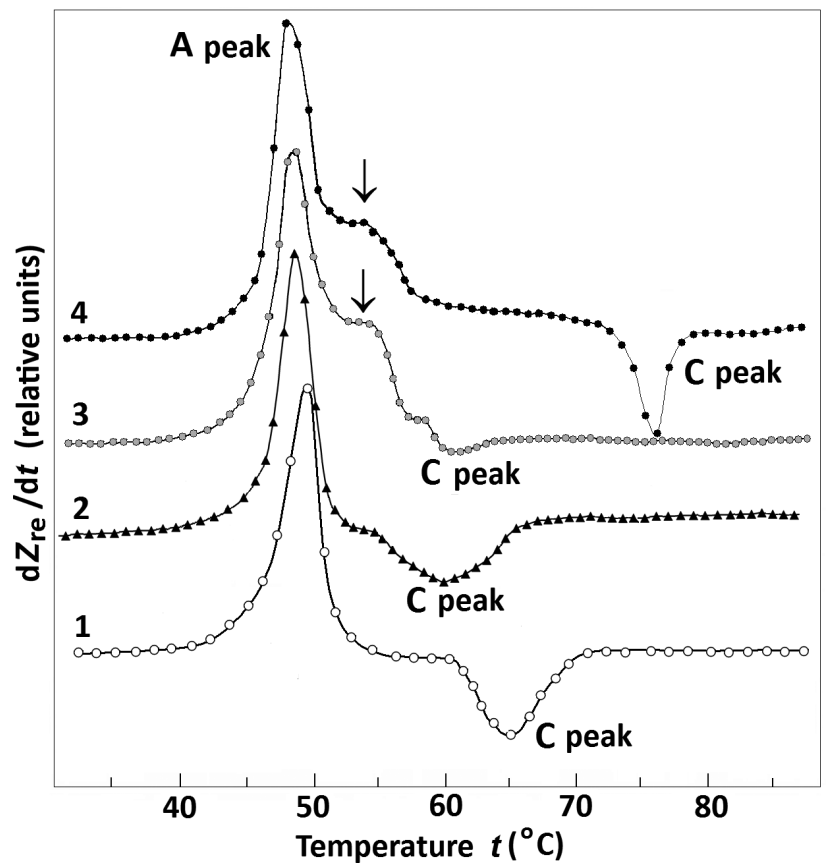

Figure 7. Effect of freeze-thaw on Triton-X-100 residues as revealed by thermal dielectroscopy. The $\mathrm{d} Z_{\mathrm{re}} / \mathrm{d} t$ is the temperature derivative of the resistance, $Z_{\mathrm{re}}$, measured at $100 \mathrm{kHz}$, of Titon-X-100 residues. Residues were prepared from EMs of intact erythrocytes (curves 1,2 and 3) and from EMs of DIDS-treated erythrocytes (curve 4). The control residues (curve 1) were not frozen, while the frozen residues were subjected to rapid thaw (curve 2) and slow thaw (curves 3 and 4). Peaks $\mathrm{A}$ and $\mathrm{C}$ exhibit the heat denaturation of spectrin and anion exchanger, respectively.

dielectric changes at $T_{\mathrm{A}}$ and $T_{\mathrm{C}}$ were used to determine the effect of freeze-thaw on respective dielectric relaxations. The effects produced by freeze-thaw on residues did not depend significantly on the value of freezing temperature $\left(-6,-16\right.$ and $\left.-80^{\circ} \mathrm{C}\right)$ and on the duration of freezing ( 1 to $10 \mathrm{~h}$ ), however, they strongly depended on the rate of thaw. When the frozen residues were rapidly thawed in a water bath at $20^{\circ} \mathrm{C}$ for 10 min the denaturation $\mathrm{C}$ peak reduced its height, broadened its half-width and preserved its area (Fig. 7, curve 2 compared to curve 1). This outcome indicated preserved number of native protein copies and reduced cooperativity of their denaturation. After slow thaw $\left(4^{\circ} \mathrm{C}\right.$ for $2 \mathrm{~h}$ ) the predominant portion of the area of $\mathrm{C}$ peak was lost (Fig. 7, curve 3 compared to curve 2) indicating strongly reduced number of native copies of band 3 in shells. The latter conclusion was confirmed by UV-spectrophotometry which detected that the amplitude of $\mathrm{C}$ peak of frozen residues was three- to four-fold reduced after slow thaw compared to rapid thaw (not shown).

The damage produced by the freeze and slow thaw on the band 3 was partially prevented by the preliminary binding of
DIDS to this protein. Compared to Triton-X-100 residues, obtained from intact EGMs and not subjected to subzero temperatures (Fig. 7, curve 1), the cooperativity and total area of the $\mathrm{C}$ peak were fully preserved in residues, obtained from DIDS-treated EGMs and subjected to freeze and slow thaw (Fig. 7, curve 4). In addition, the denaturation temperature, $T_{\mathrm{C}}$, of band 3 of latter residues was increased by $13^{\circ} \mathrm{C}$ (Fig. 7, curve 4 ), a shift characteristic to DIDS-treated EGMs (Snow et al. 1978). However, the protective effect of DIDS was partial as the relaxation frequency of band 3 in DIDS-treated residues was decreased from $1300 \mathrm{kHz}$ in residues not subjected to freeze-thaw to $350 \mathrm{kHz}$ in residues subjected to freeze-thaw (not shown). This outcome possibly indicates that freeze-thaw produced aggregation and clustering of band 3 .

Compared to band 3, spectrin was mildly damaged by the freeze-thaw of Triton-X-100 residues. The freeze-thaw cycle did not affect the cooperativity of spectrin denaturation as expressed by the preserved height and half-width of the A peak. The inducing temperature, $\mathrm{T}_{\mathrm{A}}$, of this peak was, however, slightly decreased (Fig. 7, curves 2 and 3 compared to curve 1). The relaxation frequency of dipoles associated to spectrin, $830 \mathrm{kHz}$ at $10 \mathrm{mM} \mathrm{NaCl}$, preserved its value after freeze and slow thaw of Triton-X-100 residues. This result also underlined the low cryosensitivity of spectrin, compared to that of band 3 .

In contrast to the rapid thaw (Fig. 7, curve 2), the slow thaw of residues produced a shoulder of the A peak centered at a temperature $6^{\circ} \mathrm{C}$ above $T_{\mathrm{A}}$ (Fig. 7 , indicated by arrow on the curves 3 and 4 ). The new denaturation was not affected by the preliminary stabilisation of band 3 with DIDS (Fig. 7, curve 4). The relaxation frequency of the protein involved was very low, about $200 \mathrm{kHz}$, possibly indicating the involvement of aggregated and clustered copies. This protein could be peripheral one, similar to spectrin, but its identity is not known at present.

\section{Discussion}

This study tested the structural stability of major proteins, spectrin and band 3, of Triton-X-100 shells of EGMs as represented by the temperature and cooperativity of their thermal denaturations. During the denaturations of spectrin at $49.5^{\circ} \mathrm{C}\left(T_{\mathrm{A}}\right)$ and band 3 at $66^{\circ} \mathrm{C}\left(T_{\mathrm{C}}\right)$ the capacitance, $C_{\text {re }}$, and resistance, $Z_{\text {re }}$, of the sample of Triton-X-100 shells both changed in the same direction, increasing at $T_{\mathrm{A}}$ and decreasing at $T_{\mathrm{C}}$ for any frequency within the $50 \mathrm{kHz}-10 \mathrm{MHz}$ interval. The changes in resistance appear consequent upon the changes in capacitance (dielectric polarisability) of Triton-X-100 shells and related changes in the resultant electric field. This conclusion is consistent with the above mentioned basic conception that 
each Triton-X-100 shell represented a network of proteins devoid of closed lipid bilayer surrounding them and, in contrast to intact erythrocytes and their resealed EGMs (Ivanov and Paarvanova 2016) it was fully transparent to the incident electric field with any frequency from the indicated frequency interval.

As shown in Fig. 2, a great number of tiny spheres (sphereshaped vesicles) were firmly attached to the spectrin skeleton of Triton-X-100 shells. These objects could be envisaged as liposomes (closed inside-out lipid bilayers) made up of the remainder of original lipid bilayers and band 3 copies of EGMs. We assume the attachment spots could include band 3 tetramers which avoided the solubilisation, preserved a portion of their original lipid milieu and remained strongly linked to spectrin via the ankyrin bridges of EGMs. This assumption is consistent to the result (Fig. 5) that within the entire frequency interval of $50 \mathrm{kHz}-10 \mathrm{MHz}$ the capacitance (dielectric polarizability) of Triton-X-100 shells decreased at $\mathrm{T}_{\mathrm{C}}$ indicating immobilization of dielectrically active segments of band 3 copies after the denaturation of band 3 . This decrease in polarizability could be explained with the restriction imposed by the lipid bilayer of attached liposomes on the mobility of band 3 dielectrically active segments after band 3 denaturation at $T_{\mathrm{C}}$.

By contrast, within the entire frequency interval of $50 \mathrm{kHz}-10 \mathrm{MHz}$ the capacitance (dielectric polarizability) of Triton-X-100 shells increased at $T_{\mathrm{A}}$ (Fig. 5) indicating that dielectrically active segments of spectrin increased their mobility after the spectrin denaturation. Previous study (Ivanov and Paarvanova 2016) reported that the change of the capacitance (dielectric polarizability) of intact erythocytes and impermeable EGMs at the spectrin denaturation temperature $\left(49.5^{\circ} \mathrm{C}\right)$ depended on frequency; it decreased at low frequencies $(50 \mathrm{kHz}-3 \mathrm{MHz})$ and increased at higher frequencies $(3-10 \mathrm{MHz})$. This frequency effect was explained by the adherence of a part of denatured spectrin to the encapsulating lipid bilayer resulting in partial immobilization of dielectrically active spectrin segments and hardening of erythrocyte plasma membrane. Combining the above two lines of evidence we arrive again at the basic conception that Triton-X-100 shells lacked an encapsulating lipid bilayer thus the dielectrically active segments of spectrin were free to demonstrate higher mobility in the denatured than in native state of spectrin.

Fig. 7 shows a damage of the band 3 protein as well as the spectrin-actin membrane skeleton after freezing and thawing the Triton-X-100 residues. The freeze-thaw of residues had minor effect on spectrin denaturation at $49^{\circ} \mathrm{C}$ although an additional protein denaturation appeared at $55^{\circ} \mathrm{C}$. The freeze and rapid thaw of Triton-X-100 residues resulted in a strong reduction of cooperativity of band 3 denaturation while the slow thaw completely eliminated the peak of this denaturation. The latter outcome is indicative of a substan- tial denaturation and/or detachment of band 3 copies of the residues. These effects of freeze-thaw were prevented in residues obtained from DIDS-treated EGMs supporting the view that the main damage implicated the membrane protein band 3. Additional study is needed to elucidate the possible damage induced by freeze /thaw on the band 3 linkage to membrane skeleton. In general, these findings are in line with other reports (Woelders and Malva 1998) that slow thaw is particularly damaging to cells during their recovery after cryopreservation. The above results could help design new experiments for finding optimal conditions to reduce the freeze damage to erythrocytes and other cells. Based on the similarity of the general structure of plasma membrane in erythrocytes and nucleated animal cells these results could be helpful for cryobiology, cryosurgery and the cryopreservation of cells and tissues.

Compared to deoxyribonucleic acids the sensitivity of spectrin denaturation temperature to formamide was several times higher (Ivanov 2001; Paarvanova et al. 2012). This outcome could be exploited for the design of drugs, which could be targeted to the major peripheral proteins, homologous to erythrocyte spectrin, in human and animal cells.

\section{Conclusions}

Heat and the freeze greatly affected the structural stability and dielectric properties of erythrocyte membrane skeletons, released after mild extraction of erythrocyte ghost membranes by Triton-X-100. The thermal methods applied well differentiated the thermal (structural) stability of spectrin and band 3 , and the contributions of these major proteins to the dielectric properties of Triton-X-100 shells. The results presented indicate that Triton-X-100 shells preserved the native structure of spectrin and band 3. The Triton-X-100 shells appear as a helpful model for exploration on the structure and dynamics of erythrocyte plasma membrane proteins and their change under a variety of adverse conditions, especially during freeze and slow/rapid thaw.

\section{References}

Ajmani R. S. (1997): Hypertension and hemorheology. Clin. Hemorheol. Microcirc. 17, 397-420

Alberts B., Johnson A., Lewis J., Roberts K., Walter P., Baff M. (2008): Molecular Biology of the Cell. (5th Edition), Garland Science Publishing, New York

Alper S. L. (1991): The band 3-related anion exchanger (AE) gene family. Annu. Rev. Physiol. 53, 549-564 https://doi.org/10.1146/annurev.ph.53.030191.003001

Beutler E., Lichtman M. A., Coller B. S., Kipps T. J., Seligshon U. (2000): Williams Hematology. (6th Edition), McGraw-Hill Professional, New York 
Blake R. D., Delcourt S. G. (1996): Thermodynamic effects of formamide on DNA stability. Nucleic Acids Res. 24, 2095-2103 https://doi.org/10.1093/nar/24.11.2095

Brandts J. F., Erickson L., Lysko K., Schwartz A. T., Taverna R. D. (1977): Calorimetric studies of the structural transitions of the human erythrocyte membrane. The involvement of spectrin in the A transition. Biochemistry 16, 3450-3454 https://doi.org/10.1021/bi00634a024

Cabantchik Z. I., Greger R. (1992): Chemical probes for anion transporters of mammalian cell membranes. Am. J. Physiol. 262, 803-827

Chien S. (1987): Red-cell deformability and its relevance to bloodflow. Annu. Rev. Physiol. 49, 177-192 https://doi.org/10.1146/annurev.ph.49.030187.001141

Ciana A., Achilli C., Balduini C., Minetti G. (2011): On the association of lipid rafts to the spectrin skeleton in human erythrocytes. Biochim. Biophys. Acta 1808, 183-190 https://doi.org/10.1016/j.bbamem.2010.08.019

Ciana A., Balduini C., Minetti G. (2005): Detergent-resistant membranes in human erythrocytes and their connection to the membrane-skeleton. J. Biosci. 30, 317-28 https://doi.org/10.1007/BF02703669

Delicou S., Xydaki A., Kontaxi C., Maragkos K. (2015): Disorders of the erythrocyte membrane. Ital. J. Med. 9, 323-329 https://doi.org/10.4081/itjm.2015.470

Dodge J. T., Mitchell C., Hanahan D. J. (1963): The preparation and chemical characteristics of hemoglobin-free ghosts of erythrocytes. Arch. Biochem. Biophys. 100, 119-130 https://doi.org/10.1016/0003-9861(63)90042-0

Fischer T. M., Haest C. W. M., Stoehr M., Kamp D., Deuticke B. (1978): Selective alterations of erythrocyte deformability by SH reagents. Evidence for an involvement of spectrin in membrane shear elasticity. Biochim. Biophys. Acta 510, 270-282 https://doi.org/10.1016/0005-2736(78)90027-5

Gimsa J., Ried C. (1995): Do band 3 protein conformational changes mediate shape changes of human erythrocytes. Mol. Membr. Biol. 12, 247-254 https://doi.org/10.3109/09687689509072424

Hendrich A. B., Michalak K., Bobrowska M., Kozubek A. (1991): Effect of spectrin on structure properties of lipid bilayers formed from mixtures of phospholipids. Fluorescence and microcalorimetric studies. Gen. Physiol. Biophys. 10, 333-342

Hianik T., Rybár P., Bernhardt I. (2000): Adiabatic compressibility of red blood cell membrane: influence of skeleton. Bioelectrochemistry 52, 197-201 https://doi.org/10.1016/S0302-4598(00)00102-1

Iglič A. (1997): A possible mechanism determining the stability of spiculated red blood cells. J. Biomech. 30, 35-40 https://doi.org/10.1016/S0021-9290(96)00100-5

Ivanov I. T. (2001): Rapid method for comparing the cytotoxicity of organic solvents and ability to destabilize proteins of erythrocyte membrane. Pharmazie 56, 808-809

Ivanov I. T., Brähler M., Georgieva R., Bäumler H. (2007): Role of membrane proteins in thermal damage and necrosis of red blood cells. Thermochim. Acta 456, 7-12 https://doi.org/10.1016/j.tca.2007.01.020
Ivanov I. T. (2010): Impedance spectroscopy of human erythrocyte membrane: Effect of frequency at the spectrin denaturation transition temperature. Bioelectrochemistry 78, 181-185 https://doi.org/10.1016/j.bioelechem.2009.08.010

Ivanov I. T., Paarvanova B., Slavov T. (2012): Dipole relaxation in erythrocyte membrane: involvement of spectrin skeleton. Bioelectrochemistry 88, 148-155 https://doi.org/10.1016/j.bioelechem.2012.03.005

Ivanov I. T., Paarvanova B. (2016): Dielectric relaxations on erythrocyte membrane as revealed by spectrin denaturation. Bioelectrochemistry 110, 59-68 https://doi.org/10.1016/j.bioelechem.2016.03.007

Jennings M. L., Passow H. (1979): Anion transport across the erythrocyte membrane, in situ proteolysis of band 3 protein, and cross-linking of proteolytic fragments by $4,4^{\prime}$-diisothiocyano dihydrostilbene-2,2'-disulfonate. Biochim. Biophys. Acta 554, 498-519 https://doi.org/10.1016/0005-2736(79)90387-0

Kell D. B. (1987): The principles and potential of electrical admittance spectroscopy: an introduction. In: Biosensors: Fundamenlals and Applications. (Eds. A.P.F. Turner, I. Karube, G. S. Wilson), pp. 427-468, Oxford University Press, Oxford

Klösgen B., Rümenapp C., Gleich B. (2011): Bioimpedance Spectroscopy. In: BetaSys: Systems Biology of Regulated Exocytosis in Pancreatic $\beta$-Cells. Vol. 2, Systems Biology (Eds. B. Boo $\beta$ Bavnbek, B. Klösgen, J. Larsen, F. Pociot, E. Renström), pp. 241-271, Springer Publishing Company https://doi.org/10.1007/978-1-4419-6956-9_11

Kralj-Iglič V., Svetina S., Žekž B. (1996): Shapes of bilayer vesicles with membrane embedded molecules. Eur. Biophys. J. 24, 311-321 https://doi.org/10.1007/BF00180372

Low P. S., Willardson B. M., Narla M., Rossi M., Shohet S. (1991): Contribution of the band 3-ankyrin interaction to erythrocyte membrane mechanical stability. Blood 77, 1581-1586

Lux S. E., John K. M., Karnovsky M. J. (1976): Irreversible deformation of the spectrin-actin lattice in irreversibly sickled cells. J. Clin. Invest. 58, 955-963 https://doi.org/10.1172/JCI108549

Mangeat P. H. (1988): Interaction of biological membranes with the cytoskeletal framework of living cells. Biol. Cell. 64, 261-281 https://doi.org/10.1016/0248-4900(88)90001-9

McMillan P. N., Luftig R. B. (1973): Preservation of erythrocyte ghost ultrastructure achieved by various fixatives. Proc. Nat. Acad. Sci. USA 70, 3060-3064 https://doi.org/10.1073/pnas.70.11.3060

Michalak K., Bobrowska M., Bialkowska K., Szopa J., Sikorski A. F. (1994): Interaction of erythrocyte spectrin with some nonbilayer phospholipids. Gen. Physiol. Biophys. 13, 57-62

Mohandas N., Gallagher P. G. (2008): Red cell membrane: past, present, and future. Blood 112, 3939-3948 https://doi.org/10.1182/blood-2008-07-161166

Mokken F. C., Kedaria M., Henny C. P., Hardeman M. R., Gelb A.W. (1992): The clinical importance of erythrocyte deformability, a hemorheological parameter. Ann. Hematol. 64, 113-122 https://doi.org/10.1007/BF01697397 
Mukhopadhyay R., Gerald Lim H. W., Wortis M. (2002): Echinocyte shapes: bending, stretching, and shear determine spicule shape and spacing. Biophys. J. 82, 1756-1772 https://doi.org/10.1016/S0006-3495(02)75527-6

Paarvanova B., Tacheva B., Dospatliev L., Karabaliev M., Ivanov I. (2012): Polarity index: a measure for the destabilization effect of organic solutes on erythrocyte membrane proteins. Trakia J. Sci. 10, 150-154

Poklar N., Petrovĉiĉ N., Oblak M., Vesnaver G. (1999): Thermodynamic stability of ribonuclease A in alkylurea solutions and preferential solvation changes accompanying its thermal denaturation: A calorimetric and spectroscopic study. Prot. Sci. 8, 832-840 https://doi.org/10.1110/ps.8.4.832

Reithmeier R. A. F., Chan S. L., Popov M. (1996): Structure of the erythrocyte band 3 anion exchanger. In: Handbook of Biological Physics. (Vol. 2), Transport Processes in Eukaryotic and Prokaryotic Organisms. (Eds. W. N. Konings, H. R. Kaback, J. S. Lolkema), pp. 281-309, Elsevier Science

Sharma S., Gokhale S. M. (2011): Solubility behaviour of integral proteins and glycophorins of mammalian erythrocyte membrane. Asian J. Exp. Biol. Sci. 2, 449-454

Shelby J. P., White J., Ganesan K., Rathod P. K., Chiu D. T. (2003): A microfluidic model for single-cell capillary obstruction by Plasmodium falciparum infected erythrocytes. Proc. Natl. Acad. Sci. USA 100, 14618-14622 https://doi.org/10.1073/pnas.2433968100

Simchon S., Jan K. M., Chien S. (1987): Influence of reduced redcell deformability on regional bloodflow. Am. J. Physiol. 253, 898-903

Snow J. W., Brandts J. F., Low P. S. (1978): The effects of anion transport inhibitors on the structural transitions in erythrocyte membranes. Biochim. Biophys. Acta 512, 579-591 https://doi.org/10.1016/0005-2736(78)90167-0

Tanner M. J. (1993): Molecular and cellular biology of the erythrocyte anion exchanger (AE1). Semin. Hematol. 30, 34-57

Van Dort H. M., Moriyama R., Low P. S. (1998): Effect of band 3 subunit equilibrium on the kinetics and affinity of ankyrin binding to erythrocyte membrane vesicles. J. Biol. Chem. 273, 14819-14826 https://doi.org/10.1074/jbc.273.24.14819

Waugh R. E., Agre P. (1988): Reductions of erythrocyte membrane viscoelastic coefficients reflect spectrin deficiencies in hereditary spherocytosis. J. Clin. Invest. 81, 133-141 https://doi.org/10.1172/JCI113284

Wildenauer D. B., Reuther H., Remien J. (1980): Reactions of the alkylating agent tris(2-chloroethyl)-amine with the erythrocyte membrane. Effects on shape changes of human erythrocytes and ghosts. Biochim. Biophys. Acta 603, 101-116 https://doi.org/10.1016/0005-2736(80)90394-6

Woelders H., Malva A. P. (1998): How important is the cooling rate in cryopreservation of (bull) semen, and what is its relation to thawing rate and glycerol concentration? Reprod. Dom. Anim. 33, 299-305 https://doi.org/10.1111/j.1439-0531.1998.tb01361.x

Yu J., Fischman D. A., Steck T. L. (1973): Selective solubilization of proteins and phospholipids from red blood cell membranes by nonionic detergents. J. Supramol. Struct. 1, 233-248 https://doi.org/10.1002/jss.400010308

Zwaal R. F. A., Schroit A. J. (1997): Pathophysiologic implications of membrane phospholipid asymmetry in blood cells. Blood 89, 1121-1132

Received: June 13, 2016

Final version accepted: September 21, 2016

First published online: February 2, 2017 